\title{
One-local retract and common fixed point in modular function spaces
}

\author{
Saleh Abdullah Al-Mezel ${ }^{1 *}$, Abdullah Al-Roqi ${ }^{1}$ and Mohamed Amine Khamsi ${ }^{2}$
}

\author{
* Correspondence: salmezel@kau. \\ edu.sa \\ 'Department of Mathematics, King \\ Abdulaziz University, PO Box 80203 \\ Jeddah 21589, Saudi Arabia \\ Full list of author information is \\ available at the end of the article
}

\begin{abstract}
In this paper, we introduce and study the concept of one-local retract in modular function spaces. In particular, we prove that any commutative family of $\rho$-nonexpansive mappings defined on a nonempty, $\rho$-closed and $\rho$-bounded subset of a modular function space has a common fixed point provided its convexity structure of admissible subsets is compact and normal.
\end{abstract}

MSC: Primary 47H09; Secondary 46B20, 47H10.

Keywords: convexity structure, fixed point, modular function space, nonexpansive mappings, normal structure, retract

\section{Introduction}

The purpose of this paper is to give an outline of a common fixed point theory for nonexpansive mappings defined on some subsets of modular function spaces. These spaces are natural generalizations of both function and sequence variants of many important, from applications perspective, spaces like Lebesgue, Orlicz, Musielak-Orlicz, Lorentz, Orlicz-Lorentz, Calderon-Lozanovskii spaces and many others. The current paper operates within the framework of convex function modulars. The importance for applications of nonexpansive mappings in modular function spaces consists in the richness of structure of modular function spaces, that-besides being Banach spaces (or $F$-spaces in a more general settings)-are equipped with modular equivalents of norm or metric notions, and also are equipped with almost everywhere convergence and convergence in submeasure. In many cases, particularly in applications to integral operators, approximation and fixed point results, modular type conditions are much more natural as modular type assumptions can be more easily verified than their metric or norm counterparts. There are also important results that can be proved only using the apparatus of modular function spaces. From this perspective, the fixed point theory in modular function spaces should be considered as complementary to the fixed point theory in normed spaces and in metric spaces.

The theory of contractions and nonexpansive mappings defined on convex subsets of Banach spaces has been well developed since the 1960s (see e.g. [1-6]), and generalized to other metric spaces (see e.g. [7-9]), and modular function spaces (see e.g. [10-12]).

In this paper, we invesigate the structure of the fixed point set of $\rho$-nonexpansive mappings. In particular, we introduce and investigate the concept of one-local retracts 
in the framework of modular function spaces. Then we show a common fixed point in this setting.

\section{Preliminaries}

Let $\Omega$ be a nonempty set and $\Sigma$ be a nontrivial $\sigma$-algebra of subsets of $\Omega$. Let $\mathcal{P}$ be a $\delta$-ring of subsets of $\Omega$, such that $E \cap A \in \mathcal{P}$ for any $E \in \mathcal{P}$ and $A \in \Sigma$. Let us assume that there exists an increasing sequence of sets $K_{n} \in \mathcal{P}$ such that $\Omega=\cup K_{n}$. By $\mathscr{E}$ we denote the linear space of all simple functions with supports from $\mathcal{P}$. By $\mathscr{M}_{\infty}$ we will denote the space of all extended measurable functions, i.e. all functions $f: \Omega \rightarrow[-\infty, \infty]$ such that there exists a sequence $\left\{g_{n}\right\} \subset \mathscr{E},\left|g_{n}\right| \leq|f|$ and $g_{n}(\omega) \rightarrow f(\omega)$ for all $\omega \in \Omega$ By $1_{A}$ we denote the characteristic function of the set $A$.

Definition 2.1. Let $\rho: \mathscr{M}_{\infty} \rightarrow[0, \infty]$ be a notrivial, convex and even function. We say that $\rho$ is a regular convex function pseudomodular if:

(i) $\rho(0)=0$;

(ii) $\rho$ is monotone, i.e. $|f(\omega)| \leq|g(\omega)|$ for all $\omega \in \Omega$ implies $\rho(f) \leq \rho(g)$, where $f, g \in$ $\mathscr{M}_{\infty}$;

(iii) $\rho$ is orthogonally subadditive, i.e $\rho\left(f 1_{A \cup B}\right) \leq \rho\left(f 1_{A}\right)+\rho\left(f 1_{B}\right)$ for any $A, B \in \sum$ such that $\mathrm{A} \cap \mathrm{B} \neq \varnothing, f \in \mathscr{M}$;

(iv) $\rho$ has the Fatou property, i.e. $\left|f_{n}(\omega)\right| \uparrow|f(\omega)|$ for all $\omega \in \Omega$ implies $\rho\left(f_{n}\right) \uparrow \rho(f)$, where $f \in \mathscr{M}_{\infty}$;

(v) $\rho$ is order continuous in $\mathscr{E}$, i.e. $g_{n} \in \mathscr{E}$ and $\left|g_{n}(\omega)\right| \downarrow 0$ implies $\rho\left(g_{n}\right) \downarrow 0$.

Similarly as in the case of measure spaces, we we say that a set $A \in \Sigma$ is $\rho$-null if $\rho\left(g 1_{A}\right)=0$ for every $g \in \mathscr{E}$. We say that a property holds $\rho$-almost everywhere if the exceptional set is $\rho$-null. As usual we identify any pair of measurable sets whose symmetric difference is $\rho$-null as well as any pair of measurable functions differing only on a $\rho$-null set. With this in mind we define

$$
\mathcal{M}(\Omega, \Sigma, \mathcal{P}, \rho)=\left\{f \in \mathcal{M}_{\infty} ;|f(\omega)|<\infty \rho-a . e\right\},
$$

where each $f \in \mathcal{M}(\Omega, \Sigma, \mathcal{P}, \rho)$ is actually an equivalence class of functions equal $\rho$-a.e. rather than an individual function. Where no confusion exists we will write $\mathscr{M}$ instead of $\mathcal{M}(\Omega, \Sigma, \mathcal{P}, \rho)$.

Definition 2.2. Let $\rho$ be a regular function pseudomodular.

(1) We say that $\rho$ is a regular convex function semimodular if $\rho(\alpha f)=0$ for every $\alpha>$ 0 implies $f=0 \rho$ - a.e.;

(2) We say that $\rho$ is a regular convex function modular if $\rho(f)=0$ implies $f=0 \rho$ - a.e.;

The class of all nonzero regular convex function modulars defined on $\Omega$ will be denoted by $\Re$.

Let us denote $\rho(f, E)=\rho\left(f 1_{E}\right)$ for $f \in \mathscr{M}, E \in \Sigma$. It is easy to prove that $\rho(f, E)$ is a function pseudomodular in the sense of Def. 2.1.1 in [13] (more precisely, it is a function pseudomodular with the Fatou property). Therefore, we can use all results of the standard theory of modular function spaces as per the framework defined by Kozlowski in [13-15], see also Musielak [16] for the basics of the general modular theory.

Definition 2.3. [13-15] Let $\rho$ be a convex function modular.

(a) A modular function space is the vector space $L_{\rho}(\Omega, \Sigma)$, or briefly $L_{\rho}$, defined by

$$
L_{\rho}=\{f \in \mathcal{M} ; \rho(\lambda f) \rightarrow 0 \text { as } \lambda \rightarrow 0\} .
$$


(b) The following formula defines a norm in $L_{\rho}$ (frequently called Luxemburg norm):

$$
\|f\|_{\rho}=\inf \{\alpha>0 ; \rho(f / \alpha) \leq 1\} .
$$

In the following theorem, we recall some of the properties of modular spaces that will be used later on in this paper.

Theorem 2.1. [13-15] Let $\rho \in \Re$.

(1) $L_{\rho},\|f\|_{\rho}$ is complete and the norm $\|\cdot\|_{\rho}$ is monotone w.r.t. the natural order in $\mathscr{M}$.

(2) $\left\|f_{n}\right\|_{\rho} \rightarrow 0$ if and only if $\rho\left(a f_{n}\right) \rightarrow 0$ for every $\alpha>0$.

(3) If $\rho\left(\alpha f_{n}\right) \rightarrow 0$ for an $\alpha>0$ then there exists a subsequence $\left\{g_{n}\right\}$ of $\left\{f_{n}\right\}$ such that $g_{n} \rightarrow 0 \rho$ - a.e.

(4) If $\left\{f_{n}\right\}$ converges uniformly to $f$ on a set $E \in \mathcal{P}$ then $\rho\left(\alpha\left(f_{n}-f\right), E\right) \rightarrow 0$ for every $\alpha>0$.

(5) Let $f_{n} \rightarrow f \rho$ - a.e. There exists a nondecreasing sequence of sets $H_{k} \in \mathcal{P}$ such that $H_{k} \uparrow \Omega$ and $\{f n\}$ converges uniformly to $f$ on every $H_{k}$ (Egoroff Theorem).

(6) $\rho(f) \leq \lim \inf \rho\left(f_{n}\right)$ whenever $f_{n} \rightarrow f \rho$ - a.e. (Note: this property is equivalent to the Fatou Property).

(7) Defining $L_{\rho}^{0}=\left\{f \in L_{\rho} ; \rho(f, \cdot)\right.$ is order continuous $\}$ and $E_{\rho}=\left\{f \in L_{\rho} ; \lambda f \in L_{\rho}^{0}\right.$ for every $\left.\lambda>0\right\}$ we have:

(a) $L_{\rho} \supset L_{\rho}^{0} \supset E_{\rho}$,

(b) $E_{\rho}$ has the Lebesgue property, i.e. $\rho\left(\alpha f, D_{k}\right) \rightarrow 0$ for $\alpha>0, f \in E_{\rho}$ and $D_{k} \downarrow \varnothing$.

(c) $E_{\rho}$ is the closure of $\mathscr{E}$ (in the sense of $\|\cdot\|_{\rho}$ ).

The following definition plays an important role in the theory of modular function spaces.

Definition 2.4. Let $\rho \in \mathfrak{R}$. We say that $\rho$ has the $\Delta_{2}$-property if $\sup _{n} \rho\left(2 f_{n}, D_{k}\right) \rightarrow 0$ whenever $D_{k} \downarrow \varnothing$ and $\sup _{n} \rho\left(f_{n}, D_{k}\right) \rightarrow 0$.

Theorem 2.2. Let $\rho \in \mathfrak{R}$. The following conditions are equivalent:

(a) $\rho$ has $\Delta_{2}$,

(b) $L_{\rho}^{0}$ is a linear subspace of $L_{\rho}$,

(c) $L_{\rho}=L_{\rho}^{0}=E_{\rho}$,

(d) if $\rho\left(f_{n}\right) \rightarrow 0$ then $\rho\left(2 f_{n}\right) \rightarrow 0$,

(e) if $\rho\left(\alpha f_{n}\right) \rightarrow 0$ for an $\alpha>0$ then $\left\|f_{n}\right\|_{\rho} \rightarrow 0$, i.e. the modular convergence is equivalent to the norm convergence.

The following definition is crucial throughout this paper.

Definition 2.5. Let $\rho \in \mathfrak{R}$.

(a) We say that $\left\{f_{n}\right\}$ is $\rho$-convergent to $f$ and write $f_{n} \rightarrow 0(\rho)$ if and only if $\rho\left(f_{n}-f\right) \rightarrow 0$.

(b) A sequence $\left\{f_{n}\right\}$ where $f_{n} \in L_{\rho}$ is called $\rho$-Cauchy if $\rho\left(f_{n}-f_{m}\right) \rightarrow 0$ as $n, m \rightarrow \infty$.

(c) A set $B \subset L_{\rho}$ is called $\rho$-closed if for any sequence of $f_{n} \in B$, the convergence $f_{n} \rightarrow f(\rho)$ implies that $f$ belongs to $B$.

(d) A set $B \subset L_{\rho}$ is called $\rho$-bounded if $\sup \{\rho(f-g) ; f \in B, g \in B\}<\infty$

(e) Let $f \in L_{\rho}$ and $C \subset L_{\rho}$. The $\rho$-distance between $f$ and $C$ is defined as

$$
d_{\rho}(f, C)=\inf \{\rho(f-g) ; g \in C\} .
$$


Let us note that $\rho$-convergence does not necessarily imply $\rho$-Cauchy condition. Also, $f_{n} \rightarrow f$ does not imply in general $\lambda f_{n} \rightarrow \lambda f, \lambda>1$. Using Theorem 2.1 it is not difficult to prove the following

Proposition 2.1. Let $\rho \in \Re$.

(i) $L_{\rho}$ is $\rho$-complete,

(ii) $\rho$-balls $B_{\rho}(x, r)=\left\{y \in L_{\rho} ; \rho(x-y) \leq r\right\}$ are $\rho$-closed.

The following property plays in the theory of modular function spaces a role similar to the reflexivity in Banach spaces (see e.g. [11]).

Definition 2.6. We say that $L_{\rho}$ has property $(R)$ if and only if every nonincreasing sequence $\left\{C_{n}\right\}$ of nonempty, $\rho$-bounded, $\rho$-closed, convex subsets of $L_{\rho}$ has nonempty intersection.

Throughout this paper we will need the following.

Definition 2.7. Let $\rho \in \mathfrak{R}$ and $C \subset L_{\rho}$ be nonempty.

(a) By the $\rho$-diameter of $C$, we will understand the number

$$
\delta_{\rho}(C)=\sup \{\rho(f-g) ; f, g \in C\} .
$$

The subset $C$ is said to be $\rho$-bounded whenever $\delta_{\rho}(C)<\infty$.

(b) The quantity $r_{\rho}(f, C)=\sup \{\rho(f-g) ; g \in C\}$ will be called the $\rho$-Chebyshev radius of $C$ with respect to $f$.

(c) The $\rho$-Chebyshev radius of $C$ is defined by $R_{\rho}(C)=\inf \left\{r_{\rho}(f, C) ; f \in C\right\}$.

(d) The $\rho$-Chebyshev center of $C$ is defined as the set

$$
\mathcal{C}_{\rho}(C)=\left\{f \in C ; r_{\rho}(f, C)=R_{\rho}(C)\right\} .
$$

Note that $R_{\rho}(C) \leq r_{\rho}(f, C) \leq \delta_{\rho}(C)$ for all $f \in C$ and observe that there is no reason, in general, for $\mathcal{C}_{\rho}(C)$ to be nonempty.

Let us finish this section with the modular definitions of $\rho$-nonexpansive mappings. The definitions are straightforward generalizations of their norm and metric equivalents.

Definition 2.8. Let $\rho \in \Re$ and $C \subset L_{\rho}$ be nonempty and $\rho$-closed. A mapping $T: C \rightarrow C$ is called a $\rho$-nonexpansive mapping if

$$
\rho(T(f)-T(g)) \leq \rho(f-g) \text { for any } f, g \in C .
$$

A point $f \in C$ is called a fixed point of $T$ whenever $T(f)=f$. The set of fixed point of $T$ is denoted by $\operatorname{Fix}(T)$.

\section{Penot compactness of admissible sets}

The following definition is needed.

Definition 2.9. Let $\rho \in \Re$ and $C \subset L_{\rho}$ be nonempty and $\rho$-bounded. We say that $A$ is an admissible subset of $C$ if

$$
A=\bigcap_{i \in I} B_{\rho}\left(b_{i}, r_{i}\right) \cap C,
$$

where $b_{i} \in C, r_{i} \geq 0$ and $I$ is an arbitrary index set. By $\mathcal{A}(C)$ we denote the family of all admissible subsets of $C$.

Note that if $C$ is $\rho$-bounded, then $C \in \mathcal{A}(C)$. In order to prove an analogue of Kirk's fixed point theorem [3], Penot [17] introduced the following definition. 
Definition 2.10. Let $\rho \in \Re$ and $C \subset L_{\rho}$ be nonempty.

(1) We will say that $\mathcal{A}(C)$ is $\rho$-normal if for any nonempty $A \in \mathcal{A}(C)$, which has more than one point, we have $R_{\rho}(A)<\delta_{\rho}(A)$.

(2) We will say that $\mathcal{A}(C)$ is compact if for any family $\left\{A_{\alpha}\right\}_{\alpha \in \Gamma} \subset \mathcal{A}(C)$ we have

$$
\bigcap_{\alpha \in \Gamma} A_{\alpha} \neq \emptyset,
$$

provided that $\bigcap_{\alpha \in F} A_{\alpha} \neq \emptyset$ for any finite subset $F$ of $\Gamma$.

Clearly if $\mathcal{A}\left(L_{\rho}\right)$ is compact, then $L_{\rho}$ has property $(R)$. In [18], the authors discussed the concept of uniform convexity in modular function spaces. In particular they proved that uniform convexity implies the property (R). Next, we show that uniform convexity implies compactness in the sense of Penot [17] of the family of convex sets. First, let us recall the definition of uniform convexity in modular function spaces. For more on this, the reader may consult [18].

Definition 2.11. Let $\rho \in \mathfrak{R}$.

(i) Let $r>0, \varepsilon>0$. Define

$$
D(r, \varepsilon)=\left\{(f, g) ; f, g \in L_{\rho}, \rho(f) \leq r, \rho(g) \leq r, \rho\left(\frac{f-g}{2}\right) \geq \varepsilon r\right\} .
$$

Let

$$
\delta(r, \varepsilon)=\inf \left\{1-\frac{1}{r} \rho\left(\frac{f+g}{2}\right) ;(f, g) \in D(r, \varepsilon)\right\}, \quad \text { if } D(r, \varepsilon) \neq \emptyset,
$$

and $\delta(r, \varepsilon)=1$ if $D(r, \varepsilon)=\varnothing$. We say that $\rho$ satisfies $(U C)$ if for every $r>0, \varepsilon>0, \delta(r, \varepsilon)>0$. Note, that for every $r>0, D(r, \varepsilon) \neq \varnothing$, for $\varepsilon>0$ small enough.

(ii) We say that $\rho$ satisfies (UUC) if for every $s \geq 0, \varepsilon>0$ there exists

$$
\eta(s, \varepsilon)>0
$$

depending on $s$ and $\varepsilon$ such that

$$
\delta(r, \varepsilon)>\eta(s, \varepsilon)>0 \text { for } r>s .
$$

(iii) We say that $\rho$ is Strictly Convex, (SC), if for every $f, g \in L_{\rho}$ such that $\rho(f)=\rho(g)$ and

$$
\rho\left(\frac{f+g}{2}\right)=\frac{\rho(f)+\rho(g)}{2}
$$

there holds $f=g$.

Note that in [11], the authors proved that in Orlicz spaces over a finite, atomless measure space, both conditions (UC) and (UUC) are equivalent. Typical examples of Orlicz functions that do not satisfy the $\Delta_{2}$ condition but are uniformly convex are: $\phi_{1}$ $(t)=e^{|t|}-|t|-1$ and $\varphi_{2}(t)=e^{t^{2}}-1$. In these cases, the associated modular is (UUC).

It is shown in [18], that if $\rho \in \Re$ is (UUC), then for any nonempty, convex, and $\rho$ closed $C \subset L_{\rho}$, and any $f \in L_{\rho}$ such that $d=d_{\rho}(f, C)<\infty$, there exists a unique best $\rho$-approximant of $f$ in $C$, i.e. a unique $g_{0} \in C$ such that 


$$
\rho\left(f-g_{0}\right)=d_{\rho}(f, C)=\inf \{\rho(f-g) ; g \in C\} .
$$

Moreover it is also shown in [18] that if $\rho \in \Re$ is (UUC), then for any nonincreasing sequence $\left\{C_{n}\right\}$ of nonempty, convex, and $\rho$-closed subsets of $L_{\rho}$, we have $\cap_{n} \geq{ }_{1} C_{n} \neq \varnothing$, provided there exists $f \in L_{\rho}$ such that $\sup _{n \geq 1} d_{\rho}\left(f, C_{n}\right)<\infty$. The authors in [18] did not show that such conclusion is still valid for any decreasing family. A property useful to get the compactness of the admissible subsets.

Theorem 2.3. Let $\rho \in \Re$. Assume $\rho \in \Re$ is (UUC). Let $\left\{C_{\alpha}\right\}_{\alpha \in \Gamma}$ be a decreasing family of nonempty, convex, $\rho$-closed subsets of $L_{\rho}$, where $(\Gamma, \prec)$ is upward directed. Assume that there exists $f L L_{\rho}$ such that $\sup _{\alpha \in \Gamma} d_{\rho}\left(f, C_{\alpha}\right)<\infty$. Then, $\cap_{\alpha \in \Gamma} C_{\alpha} \neq \varnothing$.

Proof. Set $d=\sup _{\alpha \in \Gamma} d_{\rho}\left(f, C_{\alpha}\right)$. Without loss of generality, we may assume $d>0$. For Any $n \geq 1$, there exists $a_{n} \in \Gamma$ such that

$$
d\left(1-\frac{1}{n}\right)<d_{\rho}\left(f, C_{\alpha_{n}}\right) \leq d .
$$

Since $(\Gamma, \prec)$ is upward directed, we may assume $\alpha_{n} \prec \alpha_{n+1}$. In particular we have $C_{\alpha_{n+1}} \subset C_{\alpha_{n}}$ for any $n \geq 1$. Since $\rho$ is (UUC), we get $C_{0}=\cap_{n \geq 1} C_{\alpha_{n}} \neq \emptyset$. Clearly $C_{0}$ is $\rho$-closed and

$$
d_{\rho}\left(f, C_{0}\right)=\sup _{n \geq 1} d_{\rho}\left(f, C_{\alpha_{n}}\right)=d .
$$

Again using the property (UUC) satisfied by $\rho$, there exists $g_{0} \in C_{0}$ unique such that $d_{\rho}\left(f, C_{0}\right)=\rho\left(f-g_{0}\right)$. Let us prove that $g_{0} \in C_{\alpha}$ for any $\alpha \in \Gamma$. Fix $\alpha \in \Gamma$. If for some $n$ $\geq 1$ we have $\alpha \prec \alpha_{n}$, then obviously we have $g_{0} \in C_{\alpha_{n}} \subset C_{\alpha}$.

Therefore let us assume that $\alpha \bigotimes \alpha_{n}$, for any $n \geq 1$. Since $\Gamma$ is upward directed, there exists $\beta_{n} \in \Gamma$ such that $\alpha_{n} \prec \beta_{n}$ and $\alpha \prec \beta_{n}$ for any $n \geq 1$. We can also assume that $\beta_{n}$ $\prec \beta_{n+1}$ for any $n \geq 1$. Again we have $C_{1}=\cap_{n \geq 1} C_{\beta_{n}} \neq \emptyset$. Since $C_{\beta_{n}} \subset C_{\alpha_{n}}$, for any $n \geq$ 1 , we get $C_{1} \subset C_{0}$. Moreover we have

$$
d=d_{\rho}\left(f, C_{0}\right) \leq d_{\rho}\left(f, C_{1}\right)=\sup _{n \geq 1} d_{\rho}\left(f, C_{\beta_{n}}\right) \leq d .
$$

Hence, $d_{\rho}\left(f, C_{1}\right)=d$ which implies the existence of a unique point $g_{1} \in C_{1}$ such that $d_{\rho}\left(f, C_{1}\right)=\rho\left(f-g_{1}\right)=d$. Since $\rho$ is uniformly convex, it must be (SC). Hence, $g_{0}=g_{1}$. In particular, we have $g_{0} \in C_{\beta_{n}}$, for any $n \geq 1$. Since $\alpha \prec \beta_{n}$, we get $C_{\beta_{n}} \subset C_{\alpha}$, for any $n \geq 1$, which implies $g_{0} \in C_{\alpha}$. Since $\alpha$ was taking arbitrary in $\Gamma$, we get $g_{0} \in \cap_{\alpha \in \Gamma} C_{\alpha}$, which implies $\cap_{\alpha \in \Gamma} C_{\alpha} \neq \varnothing$.

Since $\rho$ is convex, $\rho$-closed balls are convex. Theorem 2.3 implies the following.

Corollary 2.1. Let $\rho \in \mathfrak{R}$ and $C \subset L_{\rho}$ be nonempty, convex, $\rho$-closed, and $\rho$-bounded. Assume $\rho$ is (UUC). Then $\mathcal{A}(C)$ is compact.

Remark 2.1. Note that under the above assumptions, $\mathcal{A}(C)$ is $\rho$-normal. Indeed let $A \in \mathcal{A}(C)$ nonempty and not reduced to one point. Let $f, g \in A$ such that $f \neq g$. Then $\rho\left(\frac{f-g}{2}\right)>0$. Since $\rho$ is (UUC), there exists $\eta>0$ such that for any $h \in A$, we have 


$$
\rho\left(h-\frac{f+g}{2}\right) \leq(1-\eta) \delta_{\rho}(A) .
$$

Hence, $r_{\rho}\left(\frac{f+g}{2}, A\right) \leq(1-\eta) \delta_{\rho}(A)$, which implies $R_{\rho}(A)<\delta_{\rho}(A)$.

Finally, we state Penot's formulation of Kirk's fixed point theorem in modular function spaces. For the sake of completeness we will give its proof.

Theorem 2.4. Let $\rho \in \mathfrak{R}$ and $C \subset L_{\rho}$ be nonempty, $\rho$-closed, and $\rho$-bounded. Assume that $\mathcal{A}(C)$ is compact and $\rho$-normal. Then any $\rho$-nonexpansive $T: C \rightarrow C$ has a fixed point.

Proof. Since $C$ is $\rho$-bounded, then we have $C \in \mathcal{A}(C)$. Since $\mathcal{A}(C)$ is compact, the family $\mathcal{F}=\{A \in \mathcal{A}(C) ; T(A) \subset A\}$ has a minimal element $K$. Set

$$
K_{0}=(\cap\{A ; A \in \mathcal{A}(C) \text { and } T(K) \subset A\}) \cap K .
$$

Note that $T(K) \subset K_{0}$. This implies that $K_{0}$ is nonempty and belongs to $\mathcal{A}(C)$. Moreover since $K_{0} \subset K$, we get $T\left(K_{0}\right) \subset T(K) \subset K_{0}$. Hence $K_{0} \in \mathscr{F}$. The minimality of $K$ implies that $K=K_{0}$. Next let $f \in K$. By definition of the $\rho$-Chebyshev radius $r_{\rho}(f, K)$, we have $K \subset B_{\rho}\left(f, r_{\rho}(f, K)\right)$. Since $T$ is $\rho$-nonexpansive, we have $T(K) \subset B_{\rho}\left(T(f), r_{\rho}(f\right.$, $K)$ ). The definition of $K_{0}$ implies $K_{0} \subset B_{\rho}\left(T(f), r_{\rho}(f, K)\right)$. Since $K=K_{0}$, we get $K \subset B_{\rho}$ $\left(T(f), r_{\rho}(f, K)\right)$, which implies $r_{\rho}(T(f), K) \leq r_{\rho}(f, K)$. Fix $f \in K$ and set $r=r_{\rho}(f, K)$. We have

$$
K_{1}=\left\{g \in K ; r_{\rho}(g, K) \leq r\right\}=\bigcap_{h \in K} B_{\rho}(h, r) \cap K .
$$

Clearly, we have $T\left(K_{1}\right) \subset K_{1}$ and $K_{1} \in \mathcal{A}(C)$. Since $K$ is minimal, we get $K=K_{1}$ which implies that the $\rho$-Chebyshev radius $r_{\rho}(f, K)$ is constant. In particular, we have $r_{\rho}(f, K)=\delta_{\rho}(K)$, for any $f \in K$. Since $\mathcal{A}(C)$ is $\rho$-normal, we conclude that $K$ does not have more than one point. Therefore, $K=\{f\}$ which forces $T(f)=f$.

In the next section, we investigate the structure of the fixed point set of $\rho$-nonexpansive mappings.

\section{One-local retract subsets in modular function spaces}

Let $\rho \in \Re$ and $C \subset L_{\rho}$ be nonempty. A nonempty subset $D$ of $C$ is said to be a onelocal retract of $C$ if for every family $\left\{B_{i} ; i L I\right\}$ of $\rho$-balls centered in $D$ such that $C \cap$ $\left(\cap_{i \in I} B_{i}\right) \neq \varnothing$, it is the case that $D \cap\left(\cap_{i \in I} B_{i}\right) \neq \varnothing$. It is immediate that each $\rho$-nonexpansive retract of $L_{\rho}$ is a one-local retract (but not conversely). Recall that $D \subset C$ is a $\rho$-nonexpansive retract of $C$ if there exists a $\rho$-nonexpansive map $R: C \rightarrow D$ such that $R(f)=f$, for every $f \in D$.

The following result will shed some light on the interest generated around this concept.

Theorem 2.5. Let $\rho \in \Re$ and $C \subset L_{\rho}$ be nonempty, $\rho$-closed, and $\rho$-bounded. Assume that $\mathcal{A}(C)$ is compact and $\rho$-normal. Then for any $\rho$-nonexpansive mapping $T: C \rightarrow C$, the fixed point set Fix $(T)$ is a nonempty one-local retract of $C$.

Proof. Theorem 2.4 shows that $\operatorname{Fix}(T)$ is nonempty. Let us complete the proof by showing it is a one-local retract of $C$. Let $\left\{B_{\rho}\left(f_{i}, r_{i}\right)\right\}_{i \in I}$ be any family of $\rho$-closed balls such that $f_{i} \in \operatorname{Fix}(T)$, for any $i \in I$, and 


$$
C_{0}=C \cap\left(\bigcap_{i \in I} B_{\rho}\left(f_{i}, r_{i}\right)\right) \neq \emptyset .
$$

Let us prove that Fix $(T) \cap\left(\cap_{i \in I} B_{\rho}\left(f_{\dot{v}} r_{i}\right)\right) \neq \varnothing$. Since $\{f i\}_{i \in I} \subset \operatorname{Fix}(T)$, and $T$ is $\rho$-nonexpansive, then $T\left(C_{0}\right) \subset C_{0}$. Clearly, $C_{0} \in \mathcal{A}(C)$ and is nonempty. Then we have $\mathcal{A}\left(C_{0}\right) \subset \mathcal{A}(C)$. Therefore, $\mathcal{A}\left(C_{0}\right)$ is compact and $\rho$-normal. Theorem 2.4 will imply that $T$ has a fixed point in $C_{0}$ which will imply

$$
\operatorname{Fix}(T) \cap\left(\bigcap_{i \in I} B_{\rho}\left(f_{i}, r_{i}\right)\right) \neq \emptyset .
$$

This result gives some information to the structure of the fixed point set. To the best of our knowledge this is the first attempt done in modular function spaces. Next we discuss some properties of one-local retract subsets.

Theorem 2.6. Let $\rho \in \mathfrak{R}$ and $C \subset L_{\rho}$ be nonempty. Let $D$ be a nonempty subset of $C$. The following are equivalent.

(i) $D$ is a one-local retract of $C$.

(ii) $D$ is a $\rho$-nonexpansive retract of $D \cup\{f\}$, for every $f \in C$.

Proof. let us prove $(i) \Rightarrow(i i)$. Let $f \in C$. We may assume that $f \notin D$. In order to construct a $\rho$-nonexpansive retract $R: D \cup\{f\} \rightarrow D$, we only need to find $R(f) \in D$ such that

$$
\rho(R(f)-g) \leq \rho(f-g), \quad \text { for every } g \in D .
$$

Since $f \in \cap_{g \in D} B_{\rho}(g, \rho(f-g))$ and $f \in C$, then

$$
C \cap\left(\bigcap_{g \in D} B_{\rho}(g, \rho(f-g))\right) \neq \emptyset .
$$

Since $D$ is a one-local retract of $C$, we get

$$
D_{0}=D \cap\left(\bigcap_{g \in D} B_{\rho}(g, \rho(f-g))\right) \neq \emptyset .
$$

Any point in $D_{0}$ will work as $R(f)$.

Next, we prove that $(i i) \Rightarrow(i)$. In order to prove that $D$ is a one-local retract of $C$, let $\left\{B_{\rho}\left(f_{i}, r_{i}\right)\right\}_{i \in I}$ be any family of $\rho$-closed balls such that $f_{i} \in D$, for any $i \in I$, and

$$
C_{0}=C \cap\left(\bigcap_{i \in I} B_{\rho}\left(f_{i}, r_{i}\right)\right) \neq \emptyset .
$$

Let us prove that $D \cap\left(\cap_{i \in I} B_{\rho}\left(f_{i}, r_{i}\right)\right)=\varnothing$. Let $f \in C_{0}$. If $f \in D$, we have nothing to prove. Assume otherwise that $f \notin D$. Property (ii) implies the existence of a $\rho$-nonexpansive retract $R: D \cup\{f\} \rightarrow C$. It is easy to check that $R(f) \in D \cap\left(\cap_{i \in I} B_{\rho}\left(f_{i} r_{i}\right)\right)=\varnothing$, which completes the proof of our theorem. $\square$

The following technical lemma will be useful for the next results. 
Lemma 2.1. Let $\rho \in \Re$ and $C \subset L_{\rho}$ be nonempty, and $\rho$-bounded. Let $D$ be a nonempty one-local retract of $C$. Set $\operatorname{co}_{C}(D)=C \cap(\cap\{A ; A \in \mathcal{A}(C)$ and $D \subset A\})$. Then

(i) $r_{\rho}(f, D)=r_{\rho}\left(f, \operatorname{co}_{C}(D)\right)$, for any $f \in C$;

(ii) $R_{\rho}\left(\operatorname{coc}_{C}(D)\right)=R_{\rho}(D)$;

(iii) $\delta_{\rho}\left(\operatorname{co}_{C}(D)\right)=\delta_{\rho}(D)$.

Proof. Let us first prove $(i)$. Fix $f \in C$. Since $D \subset \operatorname{co}_{C}(D)$, we get $r_{\rho}(f, D) \leq r_{\rho}\left(f, c o_{C}\right.$ $(D))$. Set $r=r_{\rho}(f, D)$. We have $D \subset B_{\rho}(f, r) \in \mathcal{A}(C)$. The definition of $c_{C}(D)$ implies $\operatorname{co}_{C}(D) \subset B_{\rho}(f, r)$. Hence $r_{\rho}\left(f, c_{C}(D)\right) \leq r=r_{\rho}(f, D)$, which implies $r_{\rho}(f, D)=r_{\rho}\left(f, c o_{C}\right.$ $(D))$.

Next, we prove (ii). Let $f \in D$. We have $f \in \operatorname{co}_{C}(D)$. Using $(i)$, we get $r_{\rho}(f, D)=r_{\rho}(f$, $\left.\operatorname{co}_{C}(D)\right) \geq R_{\rho}\left(\operatorname{co}_{C}(D)\right)$. Hence, $R_{\rho}(D) \geq R_{\rho}\left(\operatorname{co}_{C}(D)\right)$. Next, let $f \in \operatorname{co}_{C}(D)$ and set $r=r_{\rho}(f$, $\left.\operatorname{co}_{C}(D)\right)$. We have $D \subset \operatorname{co}_{C}(D) \subset B_{\rho}(f, r)$. Hence, $f \in \cap_{g \in D} B_{\rho}(g, r)$. Hence, $C \cap\left(\cap_{g \in D} B_{\rho}\right.$ $(g, r))=\varnothing$. Since $D$ is a one-local retract of $C$, we get $D_{0}=D \cap\left(\cap_{g \in D} B_{\rho}(g, r)\right)=\varnothing$. Let $g \in D_{0}$. Then it is easy to see that $r_{\rho}(g, D) \leq r$. Hence, $R_{\rho}(D) \leq r$. Since $f$ was arbitrary taken in $\operatorname{co}_{C}(D)$, we get $R_{\rho}(D) \leq R_{\rho}\left(\operatorname{co}_{C}(D)\right)$, which implies $R_{\rho}(D)=R_{\rho}\left(\operatorname{co}_{C}(D)\right)$.

Finally, let us prove (iii). Since $D \subset \operatorname{co}_{C}(D)$, we get $\delta_{\rho}(D) \leq \delta_{\rho}\left(\operatorname{co}_{C}(D)\right)$. Next set $d=$ $\delta_{\rho}(D)$. Then, for any $f \in D$, we have $D \subset B_{\rho}(f, d)$. Hence $\operatorname{co}_{C}(D) \subset B_{\rho}(f, d)$. This implies $f \in \cap_{\left.g \in \operatorname{coc}_{C}(D)\right)} B_{\rho}(g, d)$. Sice $f$ was taken arbitrary in $D$, we get $D \subset \cap_{\left.g \in \cos _{C}(D)\right)} B_{\rho}(g, d)$. The definition of $\operatorname{co}_{C}(D)$ implies $\operatorname{co}_{C}(D) \subset \cap_{\left.g \in c_{C}(D)\right)} B_{\rho}(g, d)$. So for any $f, g \in c o_{C}(D)$, we have $\rho(f-g) \leq d$. Hence $\delta_{\rho}\left(\operatorname{co}_{C}(D)\right) \leq d=\delta_{\rho}(D)$, which implies $\delta_{\rho}(D)=\delta_{\rho}\left(\operatorname{co}_{C}(D)\right)$. 口

As an application of this lemma we get the following result.

Theorem 2.7. Let $\rho \in \Re$ and $C \subset L_{\rho}$ be nonempty, $\rho$-closed, and $\rho$-bounded. Assume that $\mathcal{A}(C)$ is compact and $\rho$-normal. If $D$ is a nonempty one-local retract of $C$, then $\mathcal{A}(D)$ is compact and $\rho$-normal.

Proof. Using the definition of one-local retract, it is easy to see that $\mathcal{A}(D)$ is compact. Let us show that $\mathcal{A}(D)$ is $\rho$-normal. Let $A_{0} \in \mathcal{A}(D)$ nonempty and not reduced to one point. Set $\operatorname{co}_{C}\left(A_{0}\right)=C \cap\left(\cap\left\{A ; A \in \mathcal{A}(C)\right.\right.$ and $\left.\left.A_{0} \subset A\right\}\right)$. Then from the Lemma 2.1, we get

$$
R_{\rho}\left(\operatorname{co}_{C}\left(A_{0}\right)\right)=R_{\rho}\left(A_{0}\right), \quad \text { and } \delta_{\rho}\left(\cos _{C}\left(A_{0}\right)\right)=\delta_{\rho}\left(A_{0}\right) .
$$

Since $\operatorname{co}_{C}\left(A_{0}\right) \in \mathcal{A}(C)$, then we must have $R_{\rho}\left(\operatorname{co}_{C}\left(A_{0}\right)\right)<\delta_{\rho}\left(\operatorname{co}_{C}\left(A_{0}\right)\right)$ because $\mathcal{A}(C)$ is $\rho$-normal. Therefore, we have $R_{\rho}\left(A_{0}\right)<\delta_{\rho}\left(A_{0}\right)$, which completes the proof of our claim. $\square$

The next result is amazing and has found many applications in metric spaces. Most of the ideas in its proof go back to Baillon's work [8].

Theorem 2.8. Let $\rho \in \Re$ and $C \subset L_{\rho}$ be nonempty, $\rho$-closed, and $\rho$-bounded. Assume that $\mathcal{A}(C)$ is compact and $\rho$-normal. Let $\left(C_{\beta}\right)_{\beta \in \Gamma}$ be a decreasing family of one-local retracts of $C$, where $(\Gamma, \prec)$ is totally ordered. Then $\cap_{\beta L \Gamma} C_{\beta}$ is not empty and is a onelocal retract of $C$.

Proof. First, let us prove that $n_{\beta\lfloor\Gamma} C_{\beta}$ is not empty. Consider the family

$$
\mathcal{F}=\left\{\prod_{\beta \in \Gamma} A_{\beta} ; A_{\beta} \in \mathcal{A}\left(C_{\beta}\right) \text { and }\left(A_{\beta}\right) \text { is decreasing }\right\} .
$$


$\mathscr{F}$ is not empty since $\prod_{\beta \in \Gamma} C_{\beta} \in \mathcal{F}$. $\mathscr{F}$ will be ordered by inclusion, i.e., $\prod_{\beta \in \Gamma} A_{\beta} \subset \prod_{\beta \in \Gamma} B_{\beta}$ if and only if $A_{\beta} \subset B_{\beta}$ for any $\beta \in \Gamma$. From Theorem 2.7, we know that $\mathcal{A}\left(C_{\beta}\right)$ is compact, for every $\beta \in \Gamma$. Therefore, $\mathscr{F}$ satisfies the hypothesis of Zorn's lemma. Hence for every $D \in \mathscr{F}$, there exists a minimal element $A \in \mathscr{F}$ such that $A \subset D$. We claim that if $A=\prod_{\beta \in \Gamma} A_{\beta}$ is minimal, then there exists $\beta_{0} \in \Gamma$ such that $\delta\left(A_{\beta}\right)=0$ for every $\beta>\beta_{0}$. Assume not, i.e., $\delta\left(A_{\beta}\right)>0$ for every $\beta \in \Gamma$. Fix $\beta \in \Gamma$. For every $K \subset$ $C$, set

$$
\begin{gathered}
\operatorname{co}_{\beta}(K)=\bigcap_{f \in C_{\beta}} B_{\rho}\left(f, r_{\rho}(f, K)\right) . \\
\text { Consider } A^{\prime}=\prod_{\alpha \in \Gamma} A_{\alpha}^{\prime} \text { where } \\
\qquad \begin{cases}A_{\alpha}^{\prime}=c_{\beta}\left(A_{\beta}\right) \cap A_{\alpha} & \text { if } \alpha \leq \beta \\
A_{\alpha}^{\prime}=A_{\alpha} & \text { if } \alpha \geq \beta .\end{cases}
\end{gathered}
$$

The family $\left(A_{\alpha \geq \beta}^{\prime}\right)$ is decreasing since $A \in \mathscr{F}$. Let $\alpha \leq \gamma \leq \beta$. Then $A_{\gamma}^{\prime} \subset A_{\alpha}^{\prime}$ since $A_{\gamma}$ $\subset A_{\alpha}$ and $A_{\beta}=c o_{\beta}\left(A_{\beta}\right) \cap A_{\beta}$. Hence the family $\left(A_{\alpha}^{\prime}\right)$ is decreasing. On the other hand if $\alpha \prec \beta$, then $\operatorname{co}_{\beta}\left(A_{\beta}\right) \cap A_{\alpha} \in \mathcal{A}\left(C_{\alpha}\right)$ since $C_{\beta} \subset C_{\alpha}$. Hence $A_{\alpha}^{\prime} \in \mathcal{A}\left(C_{\alpha}\right)$. Therefore, we have $A^{\prime} \in \mathscr{F}$. Since $A$ is minimal, then $A=A^{\prime}$. Hence

$$
A_{\alpha}=\operatorname{co}_{\beta}\left(A_{\beta}\right) \cap A_{\alpha}, \quad \text { for every } \alpha \prec \beta .
$$

Let $f \in C_{\beta}$ and $a<\beta$. Since $A_{\beta} \subset A_{\alpha}$, then $r_{\rho}\left(f, A_{\beta}\right) \leq r_{\rho}\left(f, A_{\alpha}\right)$. Because $\operatorname{co}_{\beta}\left(A_{\beta}\right)=\cap_{g \in C_{\beta}} B_{\rho}\left(g, r_{\rho}\left(g, A_{\beta}\right)\right)$, then we have $c_{\beta}\left(A_{\beta}\right) \subset B_{\rho}\left(g, r_{\rho}\left(g, A_{\beta}\right)\right)$ which implies $r_{\rho}\left(g, A_{\beta}\right) \leq r_{\rho}\left(g, A_{\alpha}\right)$. Since $A_{\alpha} \subset \operatorname{co}_{\beta}\left(A_{\beta}\right)$, then

$$
r_{\rho}\left(g, A_{\beta}\right) \leq r_{\rho}\left(g, A_{\alpha}\right) \leq r_{\rho}\left(g, c o_{\beta}\left(A_{\beta}\right)\right) \leq r_{\rho}\left(g, A_{\beta}\right) .
$$

Therefore, we have $r_{\rho}\left(g, A_{\alpha}\right) \leq r_{\rho}\left(g, A_{\beta}\right)$ for every $g \in C_{\beta}$. Using the definition of the $\rho$-Chebyshev radius $R_{\rho}$, we get

$$
R_{\rho}\left(A_{\alpha}\right) \leq R_{\rho}\left(A_{\beta}\right) .
$$

Let $f \in A_{\alpha}$ and set $s=r_{\rho}\left(f, A_{\alpha}\right)$. Then $f \in c o_{\beta}\left(A_{\beta}\right)$ since $A_{\alpha} \subset c o_{\beta}\left(A_{\beta}\right)$. Hence, $f \in\left(\cap_{g \in A_{\beta}} B_{\rho}(g, s)\right) \cap c_{\beta}\left(A_{\beta}\right)$. Since $C_{\beta}$ is a one-local retract of $C$, then

$$
S_{\beta}=C_{\beta} \cap\left(\cap_{g \in A_{\beta}} B_{\rho}(g, s)\right) \cap c_{\beta}\left(A_{\beta}\right) \neq \emptyset .
$$

Since $A_{\beta}=C_{\beta} \cap \operatorname{co}_{\beta}\left(A_{\beta}\right)$, then we have

$$
S_{\beta}=A_{\beta} \cap\left(\bigcap_{g \in A_{\beta}} B_{\rho}(g, s)\right) .
$$

Let $h \in S_{\beta}$, then $h \in \bigcap_{g \in A_{\beta}} B_{\rho}(g, s)$. Hence, $r_{\rho}\left(h, A_{\beta}\right) \leq s$ which implies $R_{\rho}\left(A_{\beta}\right) \leq s=r_{\rho}$ $\left(f, A_{\alpha}\right)$, for every $f \in A_{\alpha}$. Hence $R_{\rho}\left(A_{\beta}\right) \leq R_{\rho}\left(A_{\alpha}\right)$. Therefore we have 


$$
R_{\rho}\left(A_{\beta}\right)=R_{\rho}\left(A_{\alpha}\right), \quad \text { for every } a, \beta \in \Gamma \text {. }
$$

Since $\delta_{\rho}\left(A_{\beta}\right)>0$ for every $\beta \in \Gamma$. Set $A_{\beta}^{\prime \prime}$ to be the $\rho$-Chebyshev center of $A_{\beta}$, i.e., $A_{\beta}^{\prime \prime}=\mathcal{C}_{\rho}\left(A_{\beta}\right)$, for every $\beta \in \Gamma$. Since $R_{\rho}\left(A_{\beta}\right)=R_{\rho}\left(A_{\alpha}\right)$, for every $\alpha, \beta \in \Gamma$, then the family $\left(A_{\beta}^{\prime \prime}\right)$ is decreasing. Indeed, let $\alpha \prec \beta$ and $f \in A_{\beta}^{\prime \prime}$. Then we have $r_{\rho}\left(f, A_{\beta}\right)=R_{\rho}$ $\left(A_{\beta}\right)$. Since we proved that $r_{\rho}\left(g, A_{\beta}\right)=r_{\rho}\left(g, A_{\alpha}\right)$, for every $g \in C_{\beta}$, then

$$
r_{\rho}\left(f, A_{\alpha}\right)=r_{\rho}\left(f, A_{\beta}\right)=R_{\rho}\left(A_{\beta}\right)=R_{\rho}\left(A_{\alpha}\right),
$$

which implies that $f \in A_{\alpha}^{\prime \prime}$. Therefore, we have $A^{\prime \prime}=\prod_{\beta \in \Gamma} A_{\beta}^{\prime \prime} \in \mathcal{F}$. Since $A^{\prime \prime} \subset A$ and $A$ is minimal, we get $A=A$ ". Therefore, we have $\mathcal{C}_{\rho}\left(A_{\beta}\right)=A_{\beta}$ for every $\beta \in \Gamma$. This contradicts the fact that $\mathcal{A}\left(C_{\beta}\right)$ is normal for every $\beta \in \Gamma$. Hence there exists $\beta_{0} \in \Gamma$ such that

$$
\delta\left(A_{\beta}\right)=0, \quad \text { for every } \beta \succ \beta_{0} .
$$

The proof of our claim is therefore complete. Then we have $A_{\beta}=\{f\}$, for every $\beta>$ $\beta_{0}$. This clearly implies that $f \in \cap_{\beta \in \Gamma} C_{\beta} \neq \varnothing$. In order to complete the proof, we need to show that $S=\cap_{\beta \in \Gamma} C_{\beta}$ is a one-local retract of $C$. Let $\left(B_{i}\right)_{i \in I}$ be a family of $\rho$-balls centered in $S$ such that $\cap_{i \in I} B_{i} \neq \varnothing$. Set $D_{\beta}=\left(\cap_{i \in I} B_{i}\right) \cap C_{\beta}$, for any $\beta \in \Gamma$. Since $C_{\beta}$ is a one-local retract of $C$, and the family $\left(B_{i}\right)$ is centered in $C_{\beta}$, then $D_{\beta}$ is not empty and $D_{\beta} \in \mathcal{A}\left(C_{\beta}\right)$. Therefore, $D=\prod_{\beta \in \Gamma} D_{\beta} \in \mathcal{F}$. Let $A=\prod_{\beta \in \Gamma} A_{\beta} \subset D$ be a minimal element of $\mathscr{\mathscr { F }}$. The above proof shows that

$$
\emptyset \neq \bigcap_{\beta \in \Gamma} A_{\beta} \subset \bigcap_{\beta \in \Gamma} D_{\beta} .
$$

The proof of Theorem 2.8 is therefore complete. $\square$

The next theorem will be useful to prove the main result of the next section.

Theorem 2.9. Let $\rho \in \mathfrak{R}$ and $C \subset L_{\rho}$ be nonempty, $\rho$-closed, and $\rho$-bounded. Assume that $\mathcal{A}(C)$ is compact and $\rho$-normal. Let $\left(C_{\beta}\right)_{\beta \in \Gamma}$ be a family of one-local retracts of $C$ such that for any finite subset $I$ of $\Gamma, \cap_{\beta \in \Gamma} C_{\beta}$ is not empty and is a one-local retract of $C$. Then $n_{\beta} \in \Gamma C_{\beta}$ is not empty and is a one-local retract of $C$.

Proof. Consider the family $\mathscr{F}$ of subsets $I \subset \Gamma$ such that for any finite subset $J \subset \Gamma$ (empty or not), we have $\cap_{\alpha \in I \cup J} C_{\alpha}$ is a nonempty one-local retract of $C$. Note that $\mathscr{F}$ is not empty since any finite subset of $\Gamma$ is in $\mathscr{F}$. Using Theorem 2.8 , we can show that $\mathscr{F}$ satisfies the hypothesis of Zorn's lemma. Hence $\mathscr{F}$ has a maximal element $I \subset \Gamma$. Assume $I \neq \Gamma$. Let $\alpha \in \Gamma \backslash I$. Obviously we have $I \cup\{\alpha\} \in \mathscr{F}$. This is a clear contradiction with the maximality of $I$. Therefore we have $I=\Gamma \in \mathscr{F}$, i.e., $\cap_{\beta \in \Gamma} C_{\beta}$ is not empty and is a one-local retract of $C$.

\section{Common fixed point result}

In the previous section, we showed that under suitable conditions, any $\rho$-nonexpansive mapping has a fixed point. In this section we will discuss the existence of a fixed point common to a family of a commutative $\rho$-nonexpansive mappings. First we will need to discuss the case of finite families. 
Theorem 2.10. Let $\rho \in \Re$ and $C \subset L_{\rho}$ be nonempty, $\rho$-closed, and $\rho$-bounded. Assume that $\mathcal{A}(C)$ is compact and $\rho$-normal. Then for any finite family $\mathscr{H}=\left\{T_{1}, T_{2}, \ldots\right.$ $\left.T_{n}\right\}$ of commutative $\rho$-nonexpansive mappings defined on $C$ has a common fixed point, i.e., Fix $\left(T_{1}\right) \cap \cdots \cap \operatorname{Fix}\left(T_{\mathrm{n}}\right) \neq \varnothing$. Moreover, the set of common fixed point set, denoted $\left.\operatorname{Fix}(\mathscr{A})=\operatorname{Fix}\left(T_{1}\right)\right) \cap \cdots \cap \operatorname{Fix}\left(T_{n}\right)$, is a one local retract of $C$.

Proof. Let us first prove Theorem 2.10 for two mappings $T_{1}$ and $T_{2}$. Using Theorem 2.5, we know that $\operatorname{Fix}\left(T_{1}\right)$ is a nonempty one-local retract of $C$. Since $T_{1}$ and $T_{2}$ are commutative, then $T_{2}\left(\operatorname{Fix}\left(T_{1}\right)\right) \subset \operatorname{Fix}\left(T_{1}\right)$. Theorems 2.4 and 2.7 show that the restriction of $T_{2}$ to $\operatorname{Fix}\left(T_{1}\right)$ has a fixed point. Again Theorem 2.5 will imply that the common fixed point set $\operatorname{Fix}\left(T_{1}\right) \cap \operatorname{Fix}\left(T_{2}\right)$ is a nonempty one-local retract of $C$. Using the same argument will show that the conclusion of Theorem 2.10 is valid for any finite number of mappings. $\square$

Next we prove the main result of this section.

Theorem 2.11. Let $\rho \in \Re$ and $C \subset L_{\rho}$ be nonempty, $\rho$-closed, and $\rho$-bounded. Assume that $\mathcal{A}(C)$ is compact and $\rho$-normal. Then for any family $\mathscr{F}=\left\{T_{i} ; i \in I\right\}$ of commutative $\rho$-nonexpansive mappings defined on $C$ has a common fixed point, i.e., $\cap_{i l}$ Fix $\left(T_{\mathrm{i}}\right) \neq \varnothing$. Moreover the set of common fixed point set, denoted $\operatorname{Fix}(\mathscr{F})=\cap_{i L I} \operatorname{Fix}\left(T_{\mathrm{i}}\right)$, is a one-local retract of $C$.

Proof. Let $\Gamma=\{\beta ; \beta$ is a nonempty finite subset of $I\}$. Theorem 2.10 implies that for every $\beta \in \Gamma$, the set $F_{\beta}$ of common fixed point set of the mappings $T_{i} i \in \beta$, is a nonempty one-local retract of $C$. Clearly the family $\left(F_{\beta}\right)_{\beta \in \Gamma}$ is decreasing and satisfies the assumptions of Theorem 2.9. Therefore, we have $n_{\beta \in \Gamma} F_{\beta}$ is nonempty and is a onelocal retract of $C$. The proof of Theorem 2.11 is complete.

Using Corollary 2.1 and Remark 2.1 we get the following result.

Corollary 2.2. Let $\rho \in \Re$ and $C \subset L_{\rho}$ be nonempty, convex, $\rho$-closed, and $\rho$-bounded. Assume $\rho$ is (UUC). Then for any family $\mathscr{F}=\left\{T_{\mathrm{i}} ; i \in I\right\}$ of commutative $\rho$-nonexpansive mappings defined on $C$ has a common fixed point, i.e., $\cap_{i L I} \operatorname{Fix}\left(T_{\mathrm{i}}\right) \neq \varnothing$. Moreover the set of common fixed point set, denoted $\operatorname{Fix}(\mathscr{P})=\cap_{i L I} \operatorname{Fix}\left(T_{\mathrm{i}}\right)$, is a one-local retract of $C$.

\section{Acknowledgements}

The authors gratefully acknowledge the financial support provided by the University of Tabuk through the project of international cooperation with the University of Texas at El Paso.

Author details

'Department of Mathematics, King Abdulaziz University, PO Box 80203, Jeddah 21589, Saudi Arabia ${ }^{2}$ Department of Mathematical Sciences, The University of Texas at El Paso, El Paso, TX 79968, USA

\section{Authors' contributions}

All authors participated in the design of this work and performed equally. All authors read and approved the final manuscript.

Competing interests

The authors declare that they have no competing interests.

Received: 6 March 2012 Accepted: 5 July 2012 Published: 5 July 2012

\section{References}

1. Browder, FE: Nonexpansive nonlinear operators in a Banach space. Proc Natl Acad Sci USA. 54, 1041-1044 (1965). doi:10.1073/pnas.54.4.1041

. Gohde, D: Zum prinzip der kontraktiven abbildung. Math Nachr. 30, 251-258 (1965). doi:10.1002/mana.19650300312 Kirk, WA: A fixed point theorem for mappings which do not increase distances. Am Math Soc. 82, 1004-1006 (1965) 4. Kirk, WA: Fixed Point Theory for Nonexpansive Mappings, I and II. In Lecture Notes in Mathematics, vol. 886, pp. 485505.Springer, Berlin (1981) 
5. Goebel, K, Reich, S: Uniform Convexity, Hyperbolic Geometry, and Nonexpansive Mappings. In Series of Monographs and Textbooks in Pure and Applied Mathematics, vol. 83,Dekker, New York (1984)

6. Dhompongsa, S, Kirk, WA, Sims, B: Fixed points of uniformly lipschitzian mappings. Nonlinear Anal. 65, 762-772 (2006). doi:10.1016/j.na.2005.09.044

7. Goebel, K, Sekowski, T, Stachura, A: Uniform convexity of the hyperbolic metric and fixed points of holomorphic mappings in the Hilbert ball. Nonlinear Anal. 4, 1011-1021 (1980). doi:10.1016/0362-546X(80)90012-7

8. Baillon, JB: Nonexpansive mappings and hyperconvex spaces. Contemp Math. 72, 11-19 (1988)

9. Khamsi, MA, Kirk, WA: An Introduction to Metric Spaces and Fixed Point Theory. Wiley, New York (2001)

10. Khamsi, MA, Kozlowski, WK, Reich, S: Fixed point theory in modular function spaces. Nonlinear Anal. 14, 935-953 (1990). doi:10.1016/0362-546X(90)90111-S

11. Khamsi, MA, Kozlowski, WM, Shutao, C: Some geometrical properties and fixed point theorems in Orlicz spaces. J Math Anal Appl. 155.2, 393-412 (1991)

12. Khamsi, MA: A convexity property in Modular function spaces. Math Japonica. 44.2, 269-279 (1996)

13. Kozlowski, WM: Modular Function Spaces. In Series of Monographs and Textbooks in Pure and Applied Mathematics, vol. 122,Dekker, New York (1988)

14. Kozlowski, WM: Notes on modular function spaces I. Comment Math. 28, 91-104 (1988)

15. Kozlowski, WM: Notes on modular function spaces II. Comment Math. 28, 105-120 (1988)

16. Musielak, J: Orlicz Spaces and Modular Spaces. In Lecture Notes in Mathematics, vol. 1034,Springer, Berlin (1983)

17. Penot, JP: Fixed point theorem without convexity, Analyse Non Convexe (1977, Pau). Bull Soc Math France, Memoire.60, 129-152

18. Khamsi, MA, Kozlowski, WK: On asymptotic pointwise nonexpansive mappings in modular function spaces. J Math Anal Appl. 380, 697-708 (2011). doi:10.1016/j.jmaa.2011.03.031

doi:10.1186/1687-1812-2012-109

Cite this article as: Al-Mezel et al:: One-local retract and common fixed point in modular function spaces. Fixed

Point Theory and Applications 2012 2012:109.

\section{Submit your manuscript to a SpringerOpen ${ }^{\circ}$} journal and benefit from:

- Convenient online submission

- Rigorous peer review

- Immediate publication on acceptance

- Open access: articles freely available online

- High visibility within the field

- Retaining the copyright to your article

Submit your next manuscript at $\gg$ springeropen.com 